\title{
A brief, computerized intervention targeting error sensitivity reduces the error-related negativity
}

\author{
Alexandria Meyer ${ }^{1}$ (D) $\cdot$ Brittany Gibby $^{1} \cdot$ Karl Wissemann $^{1} \cdot$ Julia Klawohn $^{2} \cdot$ Greg Hajcak $^{1} \cdot$ Norman B. Schmidt $^{1}$
}

Published online: 9 December 2019

(C) The Psychonomic Society, Inc. 2019

\begin{abstract}
Research has identified the neural response to errors (the error-related negativity; ERN) as a marker of current anxiety, as well as risk for future anxiety. Previous work found that traditional cognitive behavioral therapy approaches do not impact the ERN. However, none of these approaches directly target the psychological constructs linked to an increased ERN (e.g., error sensitivity). In the current study, we examine the extent to which a brief, computerized intervention ("Treating the ERN"; i.e., TERN) might impact the ERN by reducing error sensitivity. Results suggest that TERN reduced the ERN and that the impact of the intervention was larger amongst individuals with an increased baseline ERN. This study is an important first step in the development of a novel intervention approach that directly targets error sensitivity, and thereby the ERN.
\end{abstract}

Keywords Error-related negativity $\cdot$ ERN $\cdot$ ERP $\cdot$ Computerized intervention

\section{Introduction}

A substantial amount of research has focused on individual differences in the error-related negativity (ERN) as a neural marker (Meyer, 2017b; Moser, Moran, Schroder, Donnellan, \& Yeung, 2013; Weinberg et al., 2016). The ERN is an eventrelated potential (ERP) that appears as a sharp, negative peak in the ERP waveform at fronto-central sites when individuals make errors on lab-based reaction-time tasks. This neural response is generated in the anterior cingulate cortex (Shackman et al., 2011) and is thought to reflect a general error-detection system in the brain. Moreover, a substantial amount of work has linked an increased ERN to individual differences in anxiety (Cavanagh \& Shackman, 2014; Hajcak, 2012; Meyer, 2016).

Errors may be conceptualized as motivationally salient, internal events that could threaten an individual's safety and require attention and corrective action. In this context, we view individual differences in the ERN as reflecting

Alexandria Meyer

ammeyer3@gmail.com

1 Department of Psychology, Florida State University, Tallahassee, FL, USA

2 Department of Clinical Psychology, Humboldt-University Berlin, Berlin, Germany variability in reactivity to errors (i.e., error sensitivity) and to an internally generated threat (Chong \& Meyer, 2018; Weinberg, Dieterich, \& Riesel, 2015). Consistent with the possibility that anxious individuals are more sensitive to such threats, the ERN has been found to be increased in anxious individuals in over 50 studies to date (Cavanagh \& Shackman, 2014; Meyer, 2017b; Weinberg et al., 2015). We, and others, have replicated this finding in individuals with obsessivecompulsive disorder (OCD; Hajcak, Franklin, Foa, \& Simons, 2008; Riesel, Endrass, Auerbach, \& Kathmann, 2015; Riesel et al., 2019), generalized anxiety disorder (GAD; Weinberg, Olvet, \& Hajcak, 2010), and social anxiety disorder (Endrass, Riesel, Kathmann, \& Buhlmann, 2014). Consistent with findings in adults, an increased ERN has also been found amongst anxious children early in development (Meyer, 2017a). Additionally, the ERN also appears to index risk for anxiety (Carrasco et al., 2013; Meyer, Danielson, et al., 2017a; Meyer, Hajcak, Torpey-Newman, Kujawa, \& Klein, 2015; Meyer, Nelson, Perlman, Klein, \& Kotov, 2018; Riesel, Endrass, Kaufmann, \& Kathmann, 2011; Riesel et al., 2019) .

While findings have consistently found an increased ERN amongst individuals with anxiety and those at risk for anxiety, less is known about what specific psychological constructs the ERN may index. Several within-subject studies suggest that the ERN reflects the salience of errors. For example, the ERN is larger when errors are more costly or significant (Ganushchak \& Schiller, 2008; Hajcak, Moser, Yeung, \& 
Simons, 2005a), when accuracy is emphasized over speed (Falkenstein, Hoormann, Christ, \& Hohnsbein, 2000; Gehring, Goss, Coles, Meyer, \& Donchin, 1993), when errors are punished (Meyer \& Gawlowska, 2017; Riesel, Weinberg, Endrass, Kathmann, \& Hajcak, 2012), and when performance is evaluated (Hajcak, Nieuwenhuis, Ridderinkhof, \& Simons, 2005b; Kim, Iwaki, Uno, \& Fujita, 2005; Meyer, Carlton, Chong, \& Wissemann, 2019).

Furthermore, work has linked the ERN to between-subject differences in perfectionism (Barke et al., 2017; PerroneMcGovern et al., 2017; Schrijvers, De Bruijn, Destoop, Hulstijn, \& Sabbe, 2010; Stahl, Acharki, Kresimon, Völler, \& Gibbons, 2015). One aspect of perfectionism is hypervigilance surrounding performance and mistakes. In fact, results from one study suggest that the ERN may be specifically related to maladaptive perfectionism (Perrone-McGovern et al., 2017). Moreover, the ERN has been linked to "doubts about actions" (i.e., the tendency to be dissatisfied with the quality of one's performance; Stahl et al., 2015). In line with these studies, the ERN has also been associated with individual differences in checking behavior (Weinberg, Kotov, \& Proudfit, 2014; Weinberg et al., 2016). Checking refers to the tendency towards self-monitoring of one's own behavior to reduce anxiety (e.g., repeatedly checking to make sure one locked the door). Furthermore, recently we found that the magnitude of the ERN relates to the degree to which individuals find mistakes aversive (i.e., error sensitivity; Chong \& Meyer, 2018). For example, individuals who are high in error sensitivity score highly on items such as: "when I notice a mistake I made, I feel upset" or "if I make a mistake, I always want to fix it." Thus, the ERN appears to index, in part, individual differences in perfectionism, concern over one's own behavior, and/or error sensitivity.

To date, four studies have investigated the ERN before and after treatment in individuals with anxiety disorders, finding that traditional cognitive behavioral therapy (CBT) approaches do not impact the ERN, despite changes in anxiety symptoms (Hajcak et al., 2008; Kujawa et al., 2016; Ladouceur et al., 2018; Riesel et al., 2015). For example, children who underwent successful exposure therapy for OCD (i.e., they no longer met criteria for OCD), still displayed an increased ERN following treatment (Hajcak et al., 2008). A recent study demonstrated that while CBT decreased anxiety symptoms, it did not impact either the ERN or worry related to performance (i.e., error sensitivity; Ladouceur et al., 2018). This suggests that the ERN and error sensitivity may remain increased in anxious individuals, even after treatment. Considering that an elevated ERN predicts risk for anxiety across development, even amongst individuals without an anxiety disorder (Meyer, Danielson, et al., 2017; Meyer et al., 2015; Meyer et al., 2018), an elevated ERN that remains in individuals post treatment may indicate risk for future increases in anxiety. These findings, taken together, suggest a need for the development of novel treatment strategies that directly target error sensitivity (and thereby the ERN) to complement existing treatment approaches.

Although studies suggest that traditional CBT approaches do not impact the magnitude of the ERN (Hajcak et al., 2008; Kujawa et al., 2016; Ladouceur et al., 2018), there is some evidence that the ERN can be modified in other ways. For example, Reinhart and Woodman (2015) showed that transcranial direct current stimulation (tDCS) may modulate ERN in the short term. Other approaches, like attention bias modification (Nelson, Jackson, Amir, \& Hajcak, 2015, 2017) and expressive writing (Schroder, Moran, \& Moser, 2018) have also been shown to impact the ERN in single sessions. Although preliminary, these studies indicate that the ERN is potentially modifiable in the context of relatively simple manipulations. However, none of these approaches directly target the psychological constructs linked to an increased ERN (e.g., error sensitivity or concern over performance or evaluation). This is particularly important insofar as recent work suggests that the link between the ERN and anxiety may be mediated by increased error sensitivity or concern over behavior (Chong \& Meyer, 2018; Meyer \& Klein, 2018), suggesting that these may be the relevant constructs to target to reduce risk for anxiety. Additionally, some of these manipulations are not ideal for young children (Rajapakse \& Kirton, 2013). Considering that an increased ERN early in development is a risk factor for the development of anxiety (Meyer, 2017a), it may be most fruitful to develop an intervention targeting error sensitivity (and thereby the ERN) that could eventually be utilized in young children. Moreover, some of these approaches are not ideal for at-home administration or widespread dissemination.

In the current study, we examine the extent to which a brief, computerized intervention that was designed to directly reduce sensitivity to errors, might impact the ERN. This intervention ("Treating the ERN"; i.e., TERN) consists of a 1-h computer-based tutorial covering topics such as perfectionism, fear of the social consequences of making a mistake, and over-valuation of the negative consequences of errors. The intervention focuses on basic concepts such as: "making mistakes is how we learn new things," "everybody makes mistakes," and "good things come from mistakes." Eighty undergraduate students were randomized to either TERN or a control condition (a computer-based presentation on healthy lifestyle choices). The ERN was measured both before and after the intervention and control conditions using a Flankers task. Based on previous work linking the ERN to perfectionism and sensitivity to errors, we hypothesized that individuals in the TERN condition would show a reduction in the magnitude of the ERN from pre to post intervention compared to individuals in the control condition. Results will potentially provide further evidence linking the psychological construct of error sensitivity to the ERN, as well as provide a first step in 
developing a novel intervention approach to reduce error sensitivity, and thereby the ERN.

\section{Method}

\section{Participants}

Participants were undergraduate students who received course credit for participation in the study. Of the 80 participants in the study, seven committed too few errors (i.e., less than six; Olvet \& Hajcak, 2009) during one of the assessments (pre or post), and were therefore removed from all further analyses. Of the remaining 73 participants included in the study, 50 were female and the mean age was 19 years. In total, 39 participants were administered the intervention condition (i.e., TERN) and 34 participants were administered the control condition. Groups (TERN vs. control) did not differ in age or gender, all $p s>.10$. All participants were given verbal and written information about the procedures of the study, and written consent was obtained. Study procedures were approved by the Institutional Review Board.

\section{Protocol}

A research assistant described all study procedures and obtained consent from undergraduate participants. Participants were randomly assigned to either the active (TERN) condition or the control condition before the lab visit. The electrodes and EEG cap were placed on the participant, after which participants completed a Flankers task (Eriksen \& Eriksen, 1974). Participants were then administered either TERN or the control computerized presentation. After completion, participants completed the Flankers task again.

\section{Treatment condition: "Treating the ERN" (TERN)}

TERN is a computerized treatment that takes approximately $1 \mathrm{~h}$ to complete. It includes both information provision as well as interactive quizzes. Participants first learn about the nature of making mistakes, the implications of making errors, and ways that people often deal with making mistakes. They are introduced to the concept of "error sensitivity," take a quiz to determine the severity of their own error sensitivity, and learn about the various ways individuals can develop a heightened sensitivity to the commission of errors. Participants are then taught about a number of common faulty beliefs held by people with elevated error sensitivity, why those beliefs occur, and how to deal with them. Participants are taught about the necessity of errors and the importance of making errors in the context of learning. Participants are presented with vignettes illustrating the ways in which elevated error sensitivity can manifest in real life and influence day-to-day decision making and behavior. In the final section of the intervention, participants are taught about a number of safety behaviors and behavioral limitations that they may engage in, and how those behaviors or limitations cause problems like maintaining anxiety and error sensitivity. Using an embedded interactive form, participants create a plan for fading their use of safety behaviors, reducing behavioral limitations, and increasing risktaking behavior (i.e., intentionally making a mistake to tolerate distress).

\section{Control condition: Physical Health Education Training (PHET)}

PHET is a computerized control condition that takes approximately $1 \mathrm{~h}$ to complete. The PHET condition is designed to be similar in length and presentation to TERN, though it focuses on the importance and benefit of a healthy lifestyle. General guidelines for achieving a healthy lifestyle are discussed, including what constitutes a proper diet, alcohol consumption, water consumption, hygiene, sleep, sexual health, stress management, life organization, social support, and the importance of a positive outlook. PHET includes audio and visual features, as well as comprehension quizzes. Furthermore, PHET includes a review of the importance of sleep, basic hygiene, and stress management on daily health.

\section{EEG task}

EEG was recorded both before and after the intervention or control computerized presentation, while participants completed an arrowhead version of the Flankers task (Eriksen \& Eriksen, 1974). On each trial, participants were shown five arrowheads and are instructed to press the left or right mouse button (depending on which direction the center arrow is pointing) as quickly as possible. There were two compatible conditions (" $<<<<<$ " and " $>>>>>$ ") and two incompatible conditions ( " $<<><<$ " and " $>><>$ "). The stimuli were presented randomly such that $50 \%$ of trials were incompatible. Stimuli were presented for $200 \mathrm{~ms}$ and the interval between the offset of one stimulus and the onset of the subsequent stimulus varied randomly between 2,300 and 2,800 ms. Participants completed a practice block of ten trials and were instructed to be both as accurate and as fast as possible. The task consisted of 11 blocks of 30 trials (330 total trials). Each block was initiated by the participant with a mouse click. To make the task as quick as possible, it terminated after participants committed 30 errors or 330 correct responses (whichever came first). The Flankers task took approximately $15 \mathrm{~min}$ to complete at both assessments. To encourage both fast and accurate responding, participants received feedback based on their performance at the end of each block. If performance was $90 \%$ correct or lower, the message "Please try to be more accurate" was displayed. If performance was above $95 \%$ 
correct, the message "Please try to respond faster" was displayed. If performance was between $90 \%$ and $95 \%$ correct, the message "You're doing a great job" was displayed.

\section{Psychophysiological recording and data analysis}

EEG was recorded continuously using an elastic cap and the ActiveTwo BioSemi system (BioSemi, Amsterdam, Netherlands). Thirty-four electrode sites were used, as well as two electrodes for the left and right mastoids. An electrooculogram (EOG) generated from eye movements and blinks was recorded using four electrodes: horizontal eye movements were measured via two electrodes placed $1 \mathrm{~cm}$ outside the outer edge of the left and right eyes. Vertical eye movements and blinks were recorded via two electrodes placed $1 \mathrm{~cm}$ above and below the right eye. The EEG signal was preamplified with a gain of one by a BioSemi ActiveTwo system. The data was digitized at a 24-bit resolution with a sampling rate of $1,024 \mathrm{~Hz}$ using a low-pass fifth-order sinc filter with a half-power cutoff of $204.8 \mathrm{~Hz}$. Each active electrode was measured online with respect to a common mode sense (CMS) active electrode producing a monopolar (nondifferential) channel. Offline, data were referenced to the average of the left and right mastoids, and band-pass filtered between 0.1 and $40 \mathrm{~Hz}$. Eye-blink and ocular corrections were conducted as per Gratton, Coles, and Donchin (1983). An automatic procedure was employed to detect and reject artifacts. The criteria applied was a voltage step of more than $50.0 \mu \mathrm{V}$ between sample points, a voltage difference of $300.0 \mu \mathrm{V}$ within a trial, and a maximum voltage difference of less than $.50 \mu \mathrm{V}$ within $100-\mathrm{ms}$ intervals. These intervals were rejected from individual channels on each trial. Overall, less than $1 \%$ of the data were excluded based on artifacts.

The EEG data were segmented for each trial beginning $500 \mathrm{~ms}$ before the response and continuing for $800 \mathrm{~ms}$ after the response. The response-locked ERPs were averaged separately for each trial type (e.g., correct and incorrect responses) to derive the correct response negativity (i.e., the $\mathrm{CRN}$ ) and the error-related negativity (i.e., the ERN). Baseline correction was performed using the interval from 500 to $-300 \mathrm{~ms}$ before response onset. Average activity between 0 and $100 \mathrm{~ms}$ at $\mathrm{Cz}$ was exported for each subject, where error-related brain activity was maximal. We utilized a regression-based method of calculating the difference between error and correct trials - the $\triangle E R N$ (Meyer, Lerner, De Los Reyes, Laird, \& Hajcak, 2017b) during each assessment (pre and post). Behavioral measures included the number of error and correct trials at each assessment. Additionally, average reaction times (RTs) on error and correct trials were calculated separately, as well as RTs on correct trials following error trials to calculate post-error RT slowing.

Statistical analyses were conducted using SPSS (Version 17.0) General Linear Model software, with Greenhouse-
Geisser correction applied to $p$ values associated with multiple-df, repeated-measures comparisons when necessitated by the violation of the assumption of sphericity. Repeatedmeasures ANOVAs were utilized to examine behavioral data (accuracy, RTs, and post-error slowing) as well as error-related brain activity. To examine the impact of the intervention (TERN vs. control) on error-related brain activity, a regression was conducted with treatment condition as the independent variable (TERN coded as 1; control coded as 2 ) and the baseline $\triangle E R N$ was included as a covariate, predicting post $\triangle \mathrm{ERN}$. The effect size of the treatment was calculated using Cohen's d, as well as $\mathrm{d}_{\mathrm{ppc} 2}$ and $\mathrm{d}_{\mathrm{Korr}}$ as outlined in Morris (2008) and Morris and Deshon (2002). Paired-samples t-tests were utilized to follow-up on treatment-related changes in error-related brain activity. We then conducted exploratory analyses to examine whether the degree of baseline ERN moderated treatment effects. To do so, we conducted a median-split on the sample based on high versus low baseline $\triangle \mathrm{ERN}$ and then conducted regression-based analyses again.

\section{Results}

\section{Behavior}

All behavioral data, including number of error and correct trials, is presented by condition (TERN vs. control) and assessment (pre vs. post) in Table 1. A repeated-measures ANOVA with number of trials (error vs. correct) and assessment (pre vs. post) was conducted, with intervention group (TERN vs. control) entered as a between-subject variable. Results suggested that there was a significant main effect of response, $F(1,72)=1104.57, p<.001$, suggesting that, overall, participants committed fewer errors than correct responses, $M=34.47, S D=11.83$, and $M=549.27, S D=$ 125.92 , respectively. However, no other main effects or interactions were significant in this model, all $p s>.20$; thus, accuracy did not differ between the pre and post assessment, nor did accuracy differ by intervention condition (TERN vs. control).

A repeated-measures ANOVA with RT (error vs. correct) and assessment (pre vs. post) was conducted, with intervention group (TERN vs. control) entered as a between-subject variable. Results suggested that there was a significant main effect of response, $F(1,71)=$ $1087.15, p<.001$, suggesting that, overall, participants were faster on error trials compared to correct trials, $M$ $=413.39, S D=82.19$, and $M=474.09, S D=63.08$, respectively. However, no other main effects or interactions were significant in this model, all $p \mathrm{~s}>.20$, suggesting that RT did not differ between the pre and post assessment, nor did RT differ by intervention condition (TERN vs. control). 
Table 1 Means and standard deviations for all main study variables (ERPs and behavioral variables) for both conditions (TERN and control) at the pre and post assessments

\begin{tabular}{|c|c|c|c|c|}
\hline & \multicolumn{2}{|l|}{ TERN $(N=39)$} & \multicolumn{2}{|l|}{ Control $(N=34)$} \\
\hline & Pre & Post & Pre & Post \\
\hline $\mathrm{CRN}$ & $9.67(6.66)$ & $9.97(6.30)$ & $8.73(6.24)$ & $9.96(5.21)$ \\
\hline ERN & $3.59(9.44)^{*}$ & $5.39(7.74)^{*}$ & $3.29(9.09)$ & $2.98(8.07)$ \\
\hline$\triangle \mathrm{ERN}$ & $-.31(7.14)^{*}$ & $1.26(5.98)^{*}$ & $.31(5.94)$ & $-1.14(7.34)$ \\
\hline Number of errors & $17.59(6.62)$ & $17.26(7.40)$ & $17.26(6.26)$ & $16.76(7.05)$ \\
\hline Number of correct & $278.90(71.43)$ & 266.08 (74.77) & $281.65(74.47)$ & $272.56(70.49)$ \\
\hline Accuracy & $92.5 \%(7.9 \%)$ & $92.8 \%(6.1 \%)$ & $92.8 \%(8.3 \%)$ & $93.1 \%(7.6 \%)$ \\
\hline RT correct & $484.60(66.52)$ & $461.01(61.35)$ & $493.73(83.01)$ & $457.50(59.11)$ \\
\hline RT error & $401.83(65.11)$ & $402.52(101.50)$ & $443.49(122.60)$ & $410.76(100.27)$ \\
\hline Post-error slowing & $15.33(31.49)$ & $14.57(34.30)$ & $6.26(31.46)$ & $15.21(33.31)$ \\
\hline
\end{tabular}

*Indicates a significant difference between the pre and post assessment based on a paired-samples $t$ test, $p<.05$

$C R N$ : correct response negativity, $E R N$ :error-related negativity, $\triangle E R N$ : $E R N$ minus $C R N, R T$ :reaction time

A repeated-measures ANOVA with post-trial RT (post-error vs. post-correct) and assessment (pre vs. post) was conducted, with intervention group (TERN vs. control) entered as a between-subject variable. Results suggested that there was a significant main effect of response, $F(1,71)=21.50, p<.001$, confirming that participants were slower following error trials compared to correct trials, $M=474.59, S D=60.36$, and $M=$ $462.25, S D=56.33$, respectively. However, no other main effects or interactions were significant in this model, all $p \mathrm{~s}>$ .20 , suggesting that post-error slowing did not differ between the pre and post assessment, nor did post-error slowing differ by intervention condition (TERN vs. control).

\section{Error-related brain activity}

All ERP variables are presented by condition (TERN vs. control) and assessment (pre vs. post) in Table 1. A repeatedmeasures ANOVA examining neural activity was conducted with response type (error vs. correct) and assessment (pre vs. post) entered as within-subject variables, and intervention group (TERN vs. control) entered as a between-subject variable. Results confirmed that the three-way interaction between response type (error vs. correct) $\times$ assessment (pre vs. post) $\times$ intervention group (TERN vs. control) was significant, $F(1$, $76)=6.72, p<.05$. Additionally, the main effect of response type (error vs. correct) was significant, $F(1,76)=34.85, p<$ .001 . However, no other main effects or two-way interactions reached significance, all $p \mathrm{~s}>.10$.

To isolate neural activity specific to error trials, a residualized difference score was calculated for both the pre and post assessments by saving the unstandardized residuals from a regression wherein the CRN was entered predicting the ERN (i.e., $\triangle E R N$; Meyer, Lerner, et al., 2017). Overall, the groups (TERN vs. control) did not differ at baseline on the ERN, $F(1,72)=.02, p=.89, \mathrm{CRN}, F(1,72)=.40, p=.53$, nor on the $\triangle \mathrm{ERN}, F(1,72)=.16, p=.69$. As an alternative statistical approach, the effect of intervention condition (TERN vs. control) on post-intervention error-related brain activity was examined using linear regression analyses. The regression was conducted with treatment condition as the independent variable (TERN coded as 1; control coded as 2). The baseline $\triangle E R N$ (calculated as a residualized difference score from a regression wherein the CRN was entered predicting the ERN) was included as a covariate. Results suggested that baseline $\triangle \mathrm{ERN}$ predicted post $\triangle \mathrm{ERN}, B=.54, S E$ $B=.10, \beta=.53, t=5.53, p<.001$. Moreover, intervention condition (TERN vs. control) predicted decreases in the $\triangle \mathrm{ERN}$ from pre to post assessment, $B=-.273, S E B=1.33$, $\beta=-.21, t=-2.06, p<.05$. The effect-size estimates of the impact of TERN on the $\triangle E R N$ were as follows: Cohen's $\mathrm{d}$ $=.48, \mathrm{~d}_{\mathrm{ppc} 2}=.45$, and $\mathrm{d}_{\mathrm{Korr}}=.46$, suggesting a medium to large effect (Morris, 2008; Morris \& DeShon, 2002). ${ }^{1}$

To further probe intervention-related changes in errorrelated brain activity, paired-samples t-tests were conducted on the ERN and CRN (pre vs. post assessment) by condition (i.e., TERN vs. control). Results suggested that amongst individuals in the control condition, neither the ERN nor the CRN differed from pre to post assessment, $t(1,33)=.19, p=.85$, and $t(1,33)=-1.17, p=.25$, respectively. However, amongst individuals in the TERN intervention condition, the CRN did not differ from pre to post assessment, $t(1,38)=-.45, p=.66$, whereas the ERN decreased in magnitude from pre to post

\footnotetext{
${ }^{1}$ Results remained consistent even when controlling for accuracy (pre and post), RTs (pre and post), and post-error slowing, all $p \mathrm{~s}<.05$, suggesting that the impact of the intervention (i.e., TERN) on the ERN was not better accounted for by changes in behavior during the task.
} 
assessment, $t(1,38)=-2.30, p<.05: M=3.09, S D=.941$ pre intervention versus $M=5.43, S D=7.57$ post intervention. These results suggest that the changes observed in the $\triangle E R N$ in the TERN intervention condition were specific to error-related brain activity.

Exploratory analyses were used to examine whether baseline $\triangle E R N$ moderated the intervention effects. To do so, we conducted a median-split on baseline $\triangle E R N$. We then conducted the same regression analyses amongst both groups that we had in the overall sample - i.e., we conducted regressions predicting post $\triangle E R N$, with pre $\triangle E R N$ and intervention group (TERN vs. control) entered as simultaneous predictors. Results suggested that amongst individuals with relatively low baseline error-related brain activity, the intervention (TERN vs. control) did not predict changes in the $\triangle \mathrm{ERN}, B=-.96, S E$ $B=1.73, \beta=-.09, t=-.56, p=.58$. However, amongst individuals with relatively high baseline error-related brain activity, the intervention (TERN vs. control) reduced the magnitude of the $\triangle \mathrm{ERN}, B=-3.32, S E B=1.76, \beta=-.31, t=-1.89$, $p=.06$, at a trend level. These results are depicted in Fig. 1 .

\section{Discussion}

Results from the current study indicate that a brief, computerized intervention targeting over-reactivity to errors can decrease the ERN. In the current study, the ERN was measured via a Flankers task before and after completing a computerized intervention or control condition. Results confirmed that individuals in the intervention condition experienced a significant reduction in the ERN, whereas individuals in the control condition showed no change in the ERN. Furthermore, the impact of the intervention on the ERN was more pronounced amongst individuals who were characterized by an increased ERN at baseline, suggesting that this intervention may be ideal for individuals high in neural reactivity to errors. This study is an important first step in the development of novel intervention approach that directly targets increased sensitivity to errors and related neural measures of error processing (i.e., the ERN).

The ERN has been shown to be increased in anxious individuals in more than 50 studies to date (Cavanagh \& Shackman, 2014; Meyer, 2017b; Weinberg et al., 2015). Moreover, in addition to indexing current anxiety, the ERN also indexes risk for anxiety (Carrasco et al., 2013; Meyer et al., 2015; Meyer et al., 2018; Riesel et al., 2015; Riesel et al., 2019), making it an ideal candidate target for preventative approaches. Results from the current study suggest that a brief, computerized intervention may be effective in reducing the magnitude of the ERN. Future work should examine to what extent changing the ERN may reduce risk for anxiety. Because an increased ERN early in development predicts future risk for anxiety (Meyer et al., 2015), it will be important for future work to extend the current intervention into early childhood to examine whether it is similarly possible to modify the
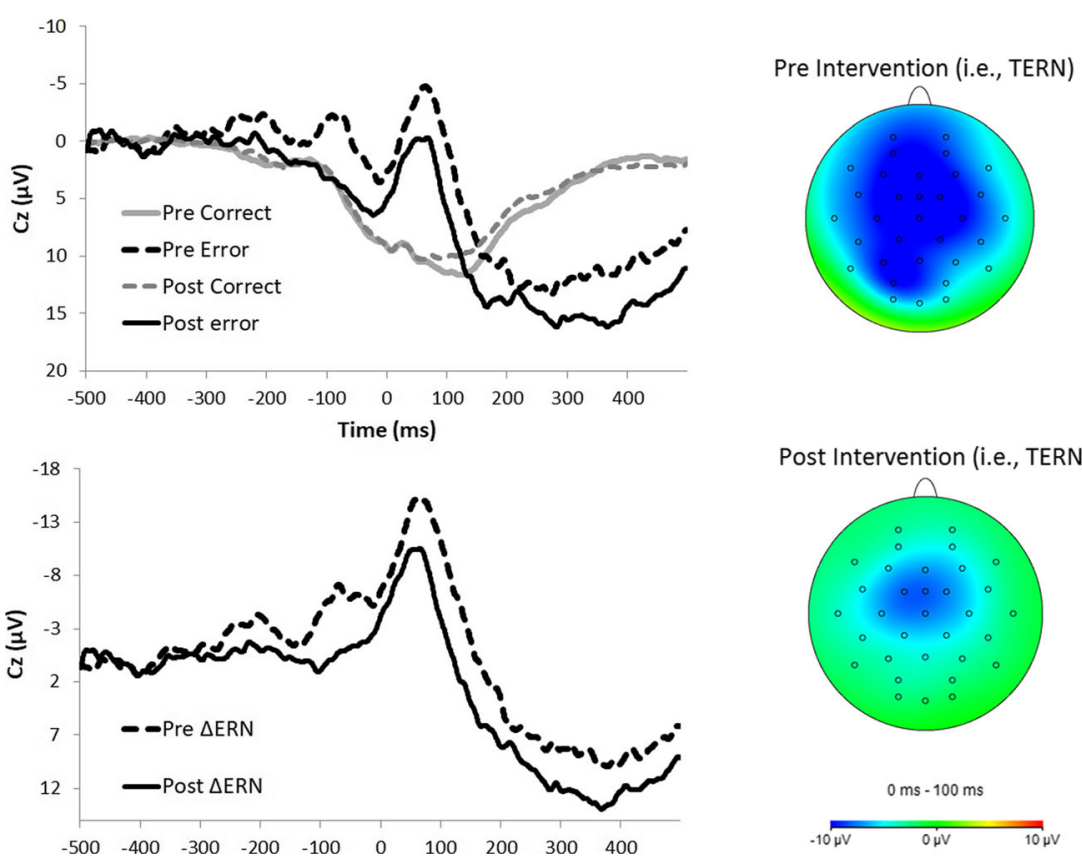

Fig. 1 On the left, waveforms at $\mathrm{Cz}$ for pre- and post-intervention (i.e., TERN) for error and correct trials (top) and the $\triangle E R N$ (error minus correct; bottom). Topographical headmaps (right) depicting neural activity for error minus correct, $0-100 \mathrm{~ms}$ after the response, for preintervention (top) and post-intervention (bottom). Participants included

had relatively large $\triangle \mathrm{ERN}$ (based on a median split) at the pre assessment. TERN appears to reduce the ERN (and $\triangle E R N$ ) amongst individuals with a large $\triangle E R N$. TERN Treating the ERN, ERN error-related negativity, $\triangle E R N$ 
ERN in young children, and if this can mitigate risk for anxiety across development.

While previous work has found that individual differences in perfectionism, checking behavior, and error sensitivity all relate to the magnitude of the ERN (Barke et al., 2017; Chong \& Meyer, 2018; Perrone-McGovern et al., 2017; Weinberg et al., 2016), this is the first study to demonstrate that by targeting error sensitivity with a psychosocial intervention, it is possible to reduce the ERN. Previous work has demonstrated that CBT approaches do not impact the ERN (Hajcak et al., 2008; Kujawa et al., 2016; Ladouceur et al., 2018). Indeed, a recent study suggested that while CBT reduced anxiety symptoms, it did not impact the ERN or worry related to performance (Ladouceur et al., 2018). These findings suggest that both the ERN and worry related to performance may remain elevated in anxious individuals, even after successful CBT treatment - leaving individuals at risk for future relapse. Future work could examine whether adding a focused intervention targeting error sensitivity (i.e., TERN) may enhance the effects of CBT on anxiety and the ERN.

It is notable that while TERN impacted error-related neural activity, it did not alter behavior during the task. Neither accuracy, reaction times, nor post-error slowing differed between the groups of individuals who received TERN vs. the control condition (i.e., PHET) at the post assessment. Given that TERN targeted emotional reactivity to, we well as the cognitive appraisal of the significance of errors (i.e., error sensitivity), we did not necessarily expect behavior to be altered during the task.

There are numerous limitations to the current study that inform future work in the area. While we view the current study as an important first step in demonstrating that the ERN can be modified via a targeted, psychosocial intervention, future work should examine if TERN relates to lasting changes in the ERN and the extent to which modifying the ERN relates to subsequent changes in anxiety symptoms or risk for anxiety. Because anxiety measures tend to assess experiences over relatively long periods (i.e., across days or weeks), the current study did not hypothesize that TERN would induce changes in standard anxiety measures, and did not include any standard assessments of anxiety symptoms before and after TERN. Therefore, we were not able to determine if individuals current or past anxiety disorders. Future work should examine the extent to which TERN may impact subsequent changes in anxiety symptoms, and whether modifying the ERN relates to longer term risk. It is possible that altering the ERN would not result in meaningful changes in anxiety symptoms or functioning. Future longitudinal work is necessary to determine whether reducing error sensitivity (and the ERN) relates to more positive outcomes.

Additionally, TERN should be compared to other intervention approaches utilized to reduce the ERN (i.e., expressive writing or ABM; Nelson et al., 2015, 2017; Schroder et al.,
2018). It is will be important to determine whether one of these intervention approaches has superior clinical utility (i.e., ability to produce lasting changes in ERN and reductions in anxiety symptoms). Relatedly, future studies might examine whether adding TERN to standard treatment approaches (i.e., CBT) could maximize outcomes in clinical populations especially among those with increased ERN.

In conclusion, the current study provides novel evidence that a brief computer-based intervention designed to reduce exaggerated error sensitivity can reduce the ERN in the shortterm. Future research will need to examine whether TERN can impact ERN over a longer follow-up period, and if ERNrelated changes predict subsequent reductions in anxiety symptoms. Furthermore, considering that the ERN indexes risk for anxiety early in development, one exciting avenue of work may be modifying TERN for young children and examining whether this approach may prevent the onset of anxiety across development.

Data availability None of the data or materials for the experiments reported here is available, and none of the experiments was preregistered.

\section{References}

Barke, A., Bode, S., Dechent, P., Schmidt-Samoa, C., Van Heer, C., \& Stahl, J. (2017). To err is (perfectly) human: behavioural and neural correlates of error processing and perfectionism. Social cognitive and affective neuroscience, 12(10), 1647-1657.

Carrasco, M., Harbin, S. M., Nienhuis, J. K., Fitzgerald, K. D., Gehring, W. J., \& Hanna, G. L. (2013). Increased Error-Related Brain Activity In Youth With Obsessive-Compulsive Disorder And Unaffected Siblingseased Error-Related Brain Activity In Youth With Obsessive-Compulsive Disorder And Unaffected Siblings. Depression and anxiety, 30(1), 39-46.

Cavanagh, J. F., \& Shackman, A. J. (2014). Frontal midline theta reflects anxiety and cognitive control: Meta-analytic evidence. Journal of Physiology-Paris.

Chong, L. J., \& Meyer, A. (2018). Understanding the Link between Anxiety and a Neural Marker of Anxiety (The Error-Related Negativity) in 5 to 7 Year-Old Children. Developmental neuropsychology, 1-17.

Endrass, T., Riesel, A., Kathmann, N., \& Buhlmann, U. (2014). Performance monitoring in obsessive-compulsive disorder and social anxiety disorder. Journal of Abnormal Psychology, 123(4), 705.

Eriksen, B., \& Eriksen, C. (1974). Effects of noise letters upon the identification of a target letter in a nonsearch task; Attention, Perception, \& Psychophysics, 16(1), 143-149. doi:https://doi.org/10.3758/ bf03203267

Falkenstein, M., Hoormann, J., Christ, S., \& Hohnsbein, J. (2000). ERP components on reaction errors and their functional significance: a tutorial. Biological psychology, 51(2-3), 87-107. doi:https://doi.org/ 10.1016/s0301-0511(99)00031-9

Ganushchak, L. Y., \& Schiller, N. O. (2008). Motivation and semantic context affect brain error-monitoring activity: an event-related brain potentials study. NeuroImage, 39(1), 395-405.

Gehring, W. J., Goss, B., Coles, M. G. H., Meyer, D. E., \& Donchin, E. (1993). A Neural System for Error Detection and Compensation. Psychological Science, 4(6), 385-390. 
Gratton, G., Coles, M. G. H., \& Donchin, E. (1983). A new method for off-line removal of ocular artifact. Electroencephalography and clinical neurophysiology, 55(4), 468-484. doi:https://doi.org/10. 1016/0013-4694(83)90135-9

Hajcak, G. (2012). What we've learned from mistakes. Current Directions in Psychological Science, 21(2), 101-106. doi:https:// doi.org/10.1177/0963721412436809

Hajcak, G., Franklin, M. E., Foa, E. B., \& Simons, R. F. (2008). Increased Error-Related Brain Activity in Pediatric Obsessive-Compulsive Disorder Before and After Treatment. Am J Psychiatry, 165(1), 116-123. doi:https://doi.org/10.1176/appi.ajp.2007.07010143

Hajcak, G., Moser, J. S., Yeung, N., \& Simons, R. F. (2005a). On the ERN and the significance of errors. Psychophysiology, 42(2), 151160

Hajcak, G., Nieuwenhuis, S., Ridderinkhof, K. R., \& Simons, R. F. (2005b). Error-preceding brain activity: robustness, temporal dynamics, and boundary conditions. Biological psychology, 70(2), 67-78.

Kim, E. Y., Iwaki, N., Uno, H., \& Fujita, T. (2005). Error-related negativity in children: effect of an observer. Developmental neuropsychology, 28(3), 871-883.

Kujawa, A., Weinberg, A., Bunford, N., Fitzgerald, K. D., Hanna, G. L., Monk, C. S., . . . Phan, K. L. (2016). Error-related brain activity in youth and young adults before and after treatment for generalized or social anxiety disorder. Progress in Neuro-Psychopharmacology and Biological Psychiatry, 71, 162-168.

Ladouceur, C. D., Tan, P. Z., Sharma, V., Bylsma, L. M., Silk, J. S., Siegle, G. J., . . . Kendall, P. C. (2018). Error-related brain activity in pediatric anxiety disorders remains elevated following individual therapy: a randomized clinical trial. Journal of Child Psychology and Psychiatry.

Meyer, A. (2016). Developing Psychiatric Biomarkers: a Review Focusing on the Error-Related Negativity as a Biomarker for Anxiety. Current Treatment Options in Psychiatry, 1-9.

Meyer, A. (2017a). A biomarker of anxiety in children and adolescents: a review focusing on the error-related negativity $(\mathrm{ERN})$ and anxiety across development. Developmental Cognitive Neuroscience, 27, 58-68.

Meyer, A. (2017b). Developing psychiatric biomarkers: a review focusing on the error-related negativity (ERN) as a biomarker for anxiety. Springer Nature.

Meyer, A., Carlton, C., Chong, L. J., \& Wissemann, K. (2019). The Presence of a Controlling Parent Is Related to an Increase in the Error-Related Negativity in 5-7 Year-Old Children. Journal of Abnormal Child Psychology, 1-11.

Meyer, A., Danielson, C. K., Danzig, A. P., Bhatia, V., Black, S. R., Bromet, E., . . . Klein, D. N. (2017a). Neural Biomarker and Early Temperament Predict Increased Internalizing Symptoms After a Natural Disaster. Journal of the American Academy of Child \& Adolescent Psychiatry, 56(5), 410-416.

Meyer, A., \& Gawlowska, M. (2017). Evidence for specificity of the impact of punishment on error-related brain activity in high versus low trait anxious individuals. International journal of psychophysiology: official journal of the International Organization of Psychophysiology.

Meyer, A., Hajcak, G., Torpey-Newman, D. C., Kujawa, A., \& Klein, D. N. (2015). Enhanced error-related brain activity in children predicts the onset of anxiety disorders between the ages of 6 and 9. Journal of Abnormal Psychology, 124(2), 266

Meyer, A., \& Klein, D. N. (2018). Examining the relationships between error-related brain activity (the ERN) and anxiety disorders versus externalizing disorders in young children: Focusing on cognitive control, fear, and shyness. Comprehensive Psychiatry, 87, 112-119.

Meyer, A., Lerner, M. D., De Los Reyes, A., Laird, R. D., \& Hajcak, G. (2017b). Considering ERP difference scores as individual difference measures: Issues with subtraction and alternative approaches. Psychophysiology, 54(1), 114-122.

Meyer, A., Nelson, B., Perlman, G., Klein, D. N., \& Kotov, R. (2018). A neural biomarker, the error-related negativity, predicts the first onset of generalized anxiety disorder in a large sample of adolescent females. Journal of Child Psychology and Psychiatry.

Morris, S. B. (2008). Estimating effect sizes from pretest-posttest-control group designs. Organizational Research Methods, 11(2), 364-386.

Morris, S. B., \& DeShon, R. P. (2002). Combining effect size estimates in meta-analysis with repeated measures and independent-groups designs. Psychological Methods, 7(1), 105.

Moser, J., Moran, T., Schroder, H., Donnellan, B., \& Yeung, N. (2013). On the relationship between anxiety and error monitoring: a metaanalysis and conceptual framework. Frontiers in human neuroscience, 7, 466.

Nelson, B. D., Jackson, F., Amir, N., \& Hajcak, G. (2015). Single-session attention bias modification and error-related brain activity. Cognitive, Affective, \& Behavioral Neuroscience, 1-11.

Nelson, B. D., Jackson, F., Amir, N., \& Hajcak, G. (2017). Attention Bias Modification Reduces Neural Correlates of Response Monitoring. Biological psychology.

Olvet, D. M., \& Hajcak, G. (2009). Reliability of error-related brain activity. Brain Research, 1284, 89-99.

Perrone-McGovern, K., Simon-Dack, S., Esche, A., Thomas, C., Beduna, K., Rider, K., . . . Matsen, J. (2017). The influence of emotional intelligence and perfectionism on Error-Related Negativity: An event related potential study. Personality and Individual Differences, 111, 65-70.

Rajapakse, T., \& Kirton, A. (2013). Non-invasive brain stimulation in children: applications and future directions. Translational neuroscience, 4(2), 217-233.

Reinhart, R. M., \& Woodman, G. F. (2015). The surprising temporal specificity of direct-current stimulation. Trends in neurosciences, 38(8), 459-461.

Riesel, A., Endrass, T., Auerbach, L. A., \& Kathmann, N. (2015). Overactive Performance Monitoring as an Endophenotype for Obsessive-Compulsive Disorder: Evidence From a Treatment Study. American Journal of Psychiatry, $0(0)$, appi.ajp.2014.14070886. doi:https://doi.org/10.1176/appi.ajp.2014. 14070886

Riesel, A., Endrass, T., Kaufmann, C., \& Kathmann, N. (2011). Overactive error-related brain activity as a candidate endophenotype for Obsessive-Compulsive Disorder: Evidence from unaffected first-degree relatives. Am J Psychiatry, 168(3), 317-324. doi: https://doi.org/10.1176/appi.ajp.2010.10030416

Riesel, A., Klawohn, J., Grützmann, R., Kaufmann, C., Heinzel, S., Bey, K., . . . Kathmann, N. (2019). Error-related brain activity as a transdiagnostic endophenotype for obsessive-compulsive disorder, anxiety and substance use disorder. Psychological medicine, 1-11.

Riesel, A., Weinberg, A., Endrass, T., Kathmann, N., \& Hajcak, G. (2012). Punishment has a lasting impact on error-related brain activity. Psychophysiology, 49(2), 239-247.

Schrijvers, D. L., De Bruijn, E. R., Destoop, M., Hulstijn, W., \& Sabbe, B. G. (2010). The impact of perfectionism and anxiety traits on action monitoring in major depressive disorder. Journal of neural transmission, 117(7), 869-880.

Schroder, H. S., Moran, T. P., \& Moser, J. S. (2018). The effect of expressive writing on the error-related negativity among individuals with chronic worry. Psychophysiology, 55(2), e12990.

Shackman, A. J., Salomons, T. V., Slagter, H. A., Fox, A. S., Winter, J. J., \& Davidson, R. J. (2011). The integration of negative affect, pain and cognitive control in the cingulate cortex. Nature Reviews Neuroscience, 12(3), 154-167.

Stahl, J., Acharki, M., Kresimon, M., Völler, F., \& Gibbons, H. (2015). Perfect error processing: Perfectionism-related variations in action 
monitoring and error processing mechanisms. International Journal of Psychophysiology, 97(2), 153-162.

Weinberg, A., Dieterich, R., \& Riesel, A. (2015). Error-related brain activity in the age of RDoC: A review of the literature. International Journal of Psychophysiology, 98(2), 276-299.

Weinberg, A., Kotov, R., \& Proudfit, G. H. (2014). Neural indicators of error processing in generalized anxiety disorder, obsessivecompulsive disorder, and major depressive disorder.

Weinberg, A., Meyer, A., Hale-Rude, E., Perlman, G., Kotov, R., Klein, D. N., \& Hajcak, G. (2016). Error-related negativity (ERN) and sustained threat: Conceptual framework and empirical evaluation in an adolescent sample. Psychophysiology, 53(3), 372-385.

Weinberg, A., Olvet, D. M., \& Hajcak, G. (2010). Increased error-related brain activity in generalized anxiety disorder. Biological psychology, 85(3), 472-480. doi:https://doi.org/10.1016/j.biopsycho.2010.09. 011

Publisher's note Springer Nature remains neutral with regard to jurisdictional claims in published maps and institutional affiliations. 